\title{
The distance to the Galactic centre based on Population II Cepheids and RR Lyrae stars ${ }^{\star}$
}

\author{
M. A. T. Groenewegen ${ }^{1}$, A. Udalski ${ }^{2}$, and G. Bono ${ }^{3,4}$ \\ 1 Instituut voor Sterrenkunde, Celestijnenlaan 200 D, 3001 Leuven, Belgium \\ e-mail: groen@ster. kuleuven . be \\ 2 Warsaw University Observatory, Aleje Ujazdowskie 4, 00-478, Warsaw, Poland \\ INAF Osservatorio Astronomico di Roma, via Frascati 33, 00040 Monte Porzio Catone, Italy \\ ${ }^{4}$ European Southern Observatory, Karl-Schwarzschild-Str. 2, 85748 Garching bei Munchen, Germany \\ Received 19 November 2007 / Accepted 11 January 2008
}

\section{ABSTRACT}

Context. The distance to the Galactic centre (GC) is important for determining the distance scale in the Universe. The value derived by Eisenhauer et al. (2005) of $7.62 \pm 0.32 \mathrm{kpc}$ based on the orbit of one star around the central black hole is shorter than most other distance estimates based on a variety of different methods.

Aims. To establish an independent distance to the GC with high accuracy, Population-II Cepheids were used that have been discovered in the OGLE-II and OGLE-III surveys.

Methods. Thirty-nine Population-II Cepheids were monitored with the SOFI infrared camera on 4 nights spanning 14 days, typically obtaining between 5 and 11 epochs of data. Light curves were fitted using the known periods from the OGLE data to determine the mean $K$-band magnitude with an accuracy of 0.01-0.02 mag. It so happens that $37 \mathrm{RR}$ Lyrae stars are in the field-of-view of the observations, and mean $K$-band magnitudes are derived for this sample as well.

Results. After correction for reddening, the period-luminosity relation of Population-II Cepheids in the $K$-band is determined, and the derived slope of $-2.24 \pm 0.14$ is consistent with the value derived by Matsunaga et al. (2006, MNRAS, 370, 1979). Fixing the slope to their more accurate value results in a zero point and implies a distance modulus to the GC of $14.51 \pm 0.12$, with an additional systematic uncertainty of $0.07 \mathrm{mag}$. Similarly, from the RR Lyrae $K$-band period-luminosity relation, we derive a value of $14.48 \pm 0.17$ (random) \pm 0.07 (syst.). The two independent determinations are averaged to find $14.50 \pm 0.10$ (random) \pm 0.07 (syst.), or $7.94 \pm 0.37 \pm 0.26 \mathrm{kpc}$. The absolute magnitude scale of the adopted period-luminosity relations is tied to an LMC distance modulus of $18.50 \pm 0.07$.

Key words. stars: distances - stars: variables: Cepheids - stars: variables: RR Lyrae - Galaxy: bulge - Galaxy: center

\section{Introduction}

The distance to astronomical objects is a crucial parameter but it is often very difficult to obtain with high precision. The distance to the Galactic centre (GC) is especially important, e.g., for dynamics (Oort constants, determining distances with a rotation model) or for calibrating standard candles. The classically accepted value comes from the review by Reid (1993) and is $R_{0}=8.0 \pm 0.5 \mathrm{kpc}$.

Over the past few years the distance to the GC based on the orbit of the star called S2 around the central black hole (BH) has got some attention. Initially, Eisenhauer et al. (2003) derived a value of $7.94 \pm 0.42 \mathrm{kpc}$, which was revised by Eisenhauer et al. (2005) to $7.62 \pm 0.32 \mathrm{kpc}$ once they had more epochs of data available. The neglect of post-Newtonian physics in these analyses may have lead to underestimating of the distance by about $0.11 \pm 0.02 \mathrm{kpc}$ (Zucker et al. 2006), then leading to a current best estimate of $7.73 \pm 0.32 \mathrm{kpc}$ (corresponding to a distance modulus $(\mathrm{DM})$ of $14.44 \pm 0.09)$ to the GC based on the $\mathrm{BH}$.

On the other hand, most other recent distance determinations give a longer distance, more in line with the classical value: (1) high-amplitude delta-scuti stars give $7.9 \pm 0.3 \mathrm{kpc}$ (McNamara et al. 2000); (2) RR Lyrae stars suggest a value

* Based on observations collected at the European Southern Observatory, Chile (ESO Programme 079.B-0107). of $8.8 \pm 0.3 \mathrm{kpc}$ (Collinge et al. 2006), $8.3 \pm 1.0 \mathrm{kpc}$ (Carney et al. 1995 ), or $8.0 \pm 0.65 \mathrm{kpc}$ (Fernley et al. 1987); (3) earlier work on the red clump (RC) gave a longer distance of $8.4 \pm 0.4 \mathrm{kpc}$ (Paczyński \& Stanek 1998), although Nishiyama et al. (2006) derive $7.52 \pm 0.10$ (stat) \pm 0.35 (syst) $\mathrm{kpc}$, and Babusiaux \& Gilmore (2005) $7.7 \pm 0.15 \mathrm{kpc}$; (4) from a comparison of Miras found in the OGLE database in the direction of the Galactic Bulge (GB) to those in the Magellanic Clouds, Groenewegen \& Blommaert (2005) find a distance in the range 8.5 to $9.0 \mathrm{kpc}$, in agreement with earlier work on Miras (Catchpole et al. 1999); (5) analysis of the Hipparcos proper motions of 220 Cepheids led to $R_{0}=8.5 \pm 0.5 \mathrm{kpc}$ (Feast \& Whitelock 1997); (6) modelling the observed colour-magnitude diagram in $V, I$, and $J, K$ using a population synthesis code, Vanhollebeke et al. (2008) derive a distance of $8.60 \pm 0.45 \mathrm{kpc}$.

With the exception of some RC based distances, the results obtained by Eisenhauer et al. imply a much shorter distance to the GC than found by most other methods, and this calls for an independent investigation of this matter.

In this paper the distance to the $\mathrm{GC}$ is determined using Population II Cepheids (hereafter P2C) discovered in the OGLE micro-lensing survey, and for which the mean $K$-band magnitude will be determined by infrared monitoring. Comparing it to the calibrated $\mathrm{P} 2 \mathrm{C}$ period-luminosity (PL) relation in the $K$ band from Matsunaga et al. (2006, hereafter M06) then provides 
the distance, after correction for reddening. In addition, the mean $K$-band magnitude will be determined for RR Lyrae stars that are in the field, and compared to the calibrated $K$-band PL-relation from Sollima et al. (2006). The Matsunaga et al. and Sollima et al. relations both imply an LMC DM of 18.50 as explained in Sect. 5.

In Sect. 2 the sample is discussed, and the observations are presented in Sect. 3 for the P2C and Sect. 3 for the RR Lyrae. The results are discussed in Sect. 5.

\section{The sample of Population II Cepheids}

Population-II Cepheids are old, low-mass stars. They are the progeny of hot $\mathrm{HB}$ stars that, after the exhaustion of core He-burning, move toward lower effective temperatures (postearly AGB), thus crossing the Cepheid instability strip. They are systematically brighter than RR Lyrae stars and have periods ranging from slightly below one to a few tens of days

Kubiak \& Udalski (2003, hereafter KU) have searched the OGLE-II database for P2C Cepheids and found 54 objects. KU determined a period-luminosity relation in the reddening-free Wesenheit index (based on $V, I$ photometry) and compared it to $\mathrm{P} 2 \mathrm{C}$ Cepheids in the LMC. The difference in DM at a typical period of $\log P=0.5$ is 3.58 . By assuming a DM for the LMC of $18.5(50.1 \mathrm{kpc})$, the distance to the GC P2C becomes $9.6 \mathrm{kpc}$ (with a substantial error bar of $1.5 \mathrm{kpc}$ ). This places the objects nominally in the Galactic Bulge (GB) region. Additional evidence is that $\mathrm{KU}$ found these objects to be located in a bar (see their Fig. 2), as for the RR Lyrae (Collinge et al. 2006), a strong indication that the $\mathrm{P} 2 \mathrm{C}$ Cepheids are indeed physically part of the GC region. These properties gave us confidence that determining individual distances to these objects would indeed accurately determine the distance to the GC.

A subset of stars was selected based on the following criteria. The $I$-band light curves of these stars were inspected and only "smooth" ones were retained because they show no, or little, effect of shocks in the atmosphere (Fig. 1 shows two examples). The range in Galactic longitude was largely restricted to $-2.5 \lesssim l \lesssim+2.5$ degrees to avoid any additional smearing in distance due to the effect of the inclination of the bar. The entire period range from 0.8 to \pm 10 days should be covered. OGLE I-band images were inspected for crowding.

In a later stage, it was also possible to extend the sample with the first results from the ongoing OGLE-III survey, and a sample of 70 new $\mathrm{P} 2 \mathrm{C}$ in the longitude range -2.5 to +2.5 degrees could be added. After inspecting the light curves and $I$-band images of these stars as well, a final combined sample of 49 stars remained, 39 of which were actually monitored. The basic properties of the sample are listed in Table 1 in the first columns: Identification, $\mathrm{RA}$, Declination, pulsation period, galactic coordinates.

\section{The observations}

The observations were carried out with the SOFI infrared camera on the $3.5 \mathrm{~m}$ NTT on ESO/La Silla in the nights of 2007, June 24, 28 and July 3, 8 in visitor mode. Photometric conditions were excellent on the second and third night with seeing as low as $0.6^{\prime \prime}$ and the telescope was actually defocused. Weather conditions were poorer on the first and last nights with seeing in the range $1.5-2^{\prime \prime}$ and cirrus and thin clouds. As the measurements of the P2C will be relative to the 2MASS objects in the field, this additional extinction did not influence this programme.

Typically we tried to observe the longest period ( $\gtrsim 7 \mathrm{~d}$ ) Cepheids at the beginning and end of the night, the intermediate
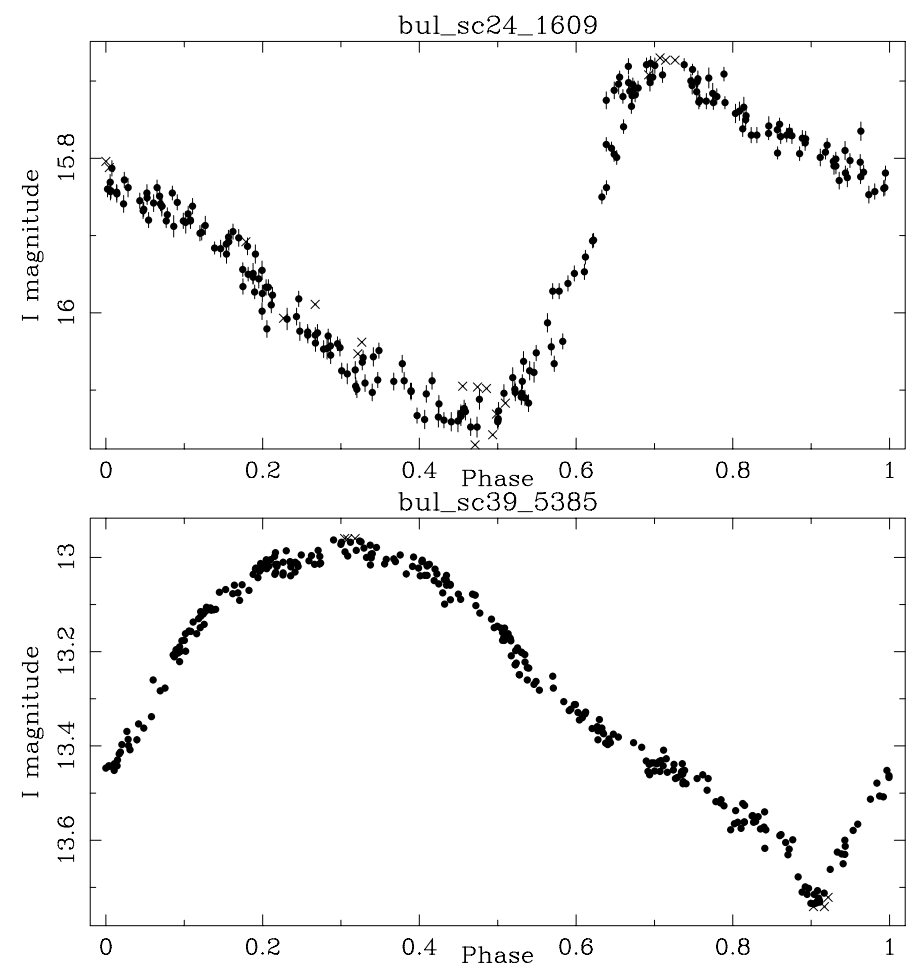

Fig. 1. Phased OGLE $I$-band light curves of two of the P2C. The periods of the two stars are 0.76522 (bul_sc24_1609) and 9.94431 (bul_sc39_5385) days.

period ones ( $\gtrsim 3 \mathrm{~d}$ ) three times per night, and the shorter period ones were observed one after the other over the entire night. In total we obtained 362 epochs of data of 39 P2C.

Images in the $K_{\mathrm{s}}$ band (hereafter simply $K$-band) were taken with the shortest possible on-chip integration time of DIT $=1.2 \mathrm{~s}$ using a pixelscale of $0.288^{\prime \prime}$ and resulting in a field-of-view of almost $5 \times 5 \operatorname{arcmin}^{1}$. The "auto-jitter" observing block (OB) was used with 9-13 exposures. The relative large number was used to have sufficient redundancy for creating a sky image in these relatively crowded fields. The $K$-band was chosen for the observations as the dispersion in the $P L$-relation is smaller in that band than in $J$ or $H$ (M06).

The data were reduced with the newly released SOFI data reduction pipeline ${ }^{2}$. The pipeline takes into account the crosstalk, the flat-field (dome flats taken with a special OB), bad pixel cleaning, and image correlation, and reconstruction.

The reduced images were trimmed to the original $1024 \times$ 1024 pixel size to eliminate the under-exposed edges. The astrometric solution was found using the WCSTools suite ${ }^{3}$ matching the stars in the field against the 2MASS catalog. Typically several tens of 2MASS objects are available and the rms in the solution typically is $0.2^{\prime \prime}$. Source extraction and PSF photometry was done using version 1.3 of DoPhot (Schechter et al. 1993). A dedicated Fortran program was written to match the 2MASS objects with the sources in the field and to determine the offset between the instrumental magnitude and the 2MASS $K_{\mathrm{s}}$-band magnitude. The brightest and faintest 2MASS objects were excluded to avoid problems of saturation in the SOFI images and

\footnotetext{
1 The $0.144^{\prime \prime}$ pixel scale was unfortunately no longer offered.

2 Available at http://www.eso.org/sci/data-processing/ software/pipelines/

3 Available at http://tdc-www.harvard.edu/software/ wcstools/
} 
bul24_1609_Ks_54276.49287.obj
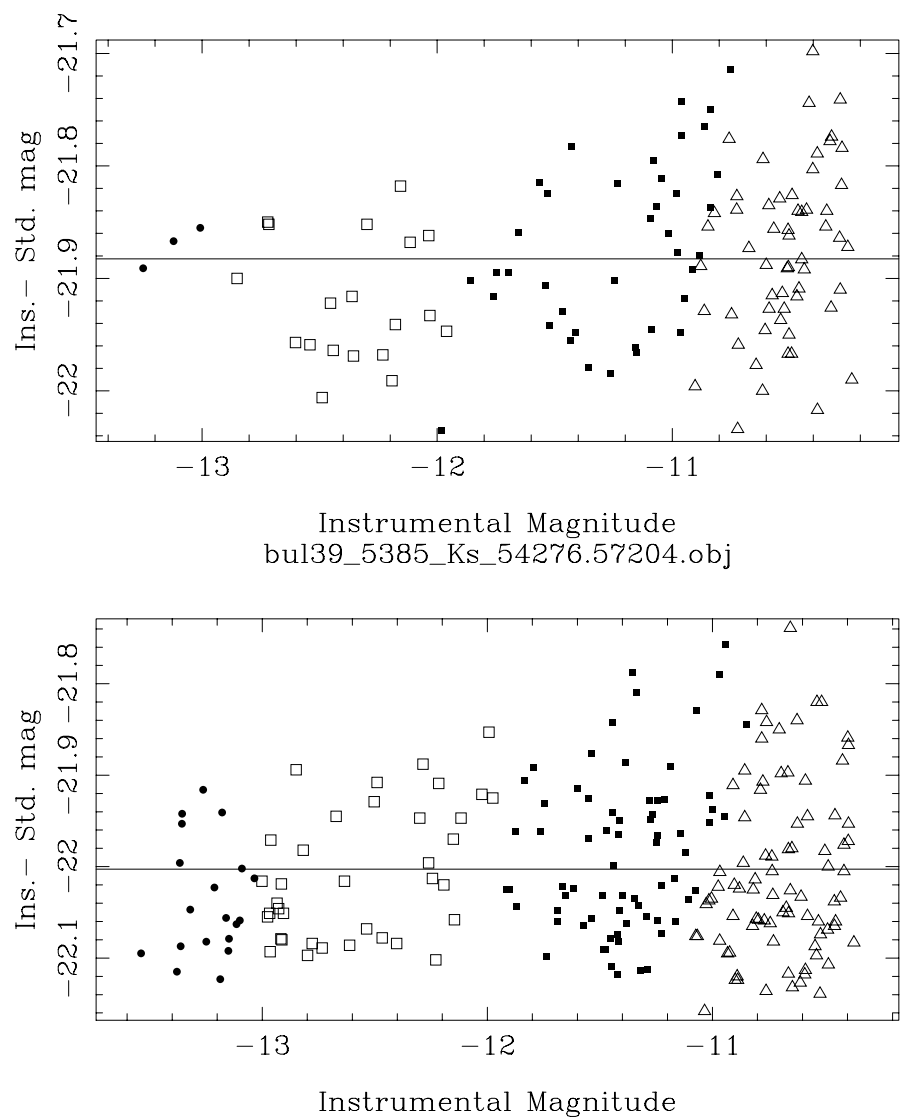

Fig. 2. The difference between SOFI instrumental magnitude and 2MASS magnitude versus instrumental magnitude for a single epoch for two different objects. The symbols indicate different 1 mag bins in 2MASS magnitude.

poor quality 2MASS data. A straight line was fitted to the data, and this determined the offset to be applied to the SOFI instrumental magnitudes to put them on the 2MASS system (an example is shown in Fig. 2). The typical error in determining this offset is $0.006 \mathrm{mag}$, and this is added in quadrature to the error in the instrumental magnitude for each star to give the total error in the observed magnitude. This error is verified by comparing the magnitudes over the different epochs for field stars and estimating the precision with which the mean magnitude can be determined.

After having determined the photometric offsets, the light curves of the P2C could be constructed. The light curves were then fitted with a sine-function with the known period from the OGLE data, and this allowed determination of the mean $K$-magnitude with high precision. Figure 3 shows the (phased) light curve for two P2C Cepheids with a short and a long period in the sample. The last three columns of Table 1 list the mean $K$-band magnitude, the precision in this determination, and the number of epochs.

\section{RR Lyrae stars}

Collinge et al. (2006) present a catalog of 1888 fundamentalmode RR Lyrae stars in the OGLE fields. It was verified which of them happen to be located in the SOFI fields of view, and for those the $K$-band light curve was extracted and fitted with a sine-curve with the known period. Table 2 presents the results, with Cols. 2-4 taken from Collinge et al. Column 5 lists in which $\mathrm{P} 2 \mathrm{C}$ field it is located, and in the analysis the corresponding reddening for that field is taken (see below). The last columns list the mean magnitude, the error, and the number of epochs. Figure 4 shows the light curve for 2 stars.

\section{Analysis}

\subsection{P2C: K-band PL-relation}

The $K$-band magnitudes are de-reddened using the model by Marshall et al. (2006). They present 3-dimensional, $K_{\mathrm{s}}$-band extinction along 64000 lines of sight at a resolution of $15^{\prime}$ typically in four distance bins. The available data within $20^{\prime}$ radius of the targets were retrieved using VizieR ${ }^{4}$. The mean and dispersion were determined for the $A_{\mathrm{K}}$ values in the bin that corresponded to a distance greater than $4 \mathrm{kpc}$ (for 2 stars a smaller distance had to be adopted). Table 1 lists the mean, dispersion, and the number of data points used in Cols. 7-9. Columns 10 of this table list the $A_{\mathrm{K}}$ value derived by multiplying the $A_{\mathrm{V}}$ value for the OGLE-II GB fields by Sumi (2004) by 0.12 (based on the reddening law of Cardelli et al. 1989).

Based on the MACHO survey towards the GB, Popowski et al. (2003) derive visual extinction for 9717 elements at a resolution of about $4^{\prime}$. The available data within $5^{\prime}$ radius of the targets was retrieved. The mean and dispersion were determined and multiplied by 0.12 to obtain the $K$-band extinction, which is listed with the number of elements used as Cols. 11-13 in Table 1.

The comparison of the reddenings based on the Marshall et al. model and the values from Sumi and Popowski et al. suggest that there are no systematic effects and that the error in the adopted reddening is realistic.

The observed $K$-magnitudes are then de-reddened, and Fig. 5 shows the observed period-luminosity relation in the $K$-band for the $\mathrm{P} 2 \mathrm{C}$, together with the best-fitting line (excluding the cross), $K_{0}=(-2.24 \pm 0.14)(\log P-1.2)+(10.578 \pm 0.099)$, with an rms of 0.41 mag. This is based on a weighted least-square fit, where the error in $A_{\mathrm{K}}$ is added in quadrature to the error in $K$. Eliminating the possible three outliers near a period of 1.3 days gives a slope of $-2.41 \pm 0.08$ and a $\mathrm{ZP}$ of $10.529 \pm 0.059$ with an rms of $0.28 \mathrm{mag}$. This dispersion is largely due to the intrinsic depth of the GB which is of the order of $1 \mathrm{kpc}$ (e.g. Babusiaux \& Gilmore 2005), and it has already been seen in the dispersion around the Wesenheit $P L$-relation by KU.

Plotting the residuals versus galactic longitude and latitude suggested a slight dependence on these parameters, and a general linear fit was made (excluding only one object):

$$
\begin{aligned}
K_{0}= & (-2.24 \pm 0.13)(\log P-1.2)+(10.60 \pm 0.17) \\
& +(-0.028 \pm 0.031) l+(0.005 \pm 0.031) b
\end{aligned}
$$

The slope agrees within the error bar with that of the $P L$-relation derived by M06, which is also on the 2 MASS system: $M_{K_{0}}=$ $(-2.41 \pm 0.05)(\log P-1.2)+(-4.00 \pm 0.02)$ with a dispersion of 0.14 mag.

To derive the distance to the GC, a Monte-Carlo simulation was performed. Data sets with new values for $A_{\mathrm{K}}$ and $K$ were generated based on the mean values and (Gaussian) errors listed in Table 1, and unweighted fits were made for a fixed slope of -2.41 . This results in a zero point (ZP) of $10.512 \pm 0.013$. The DM to the GC is therefore $14.51 \pm 0.02$, corresponding

${ }^{4}$ http://vizier.u-strasbg.fr/viz-bin/VizieR 
bul24_1609

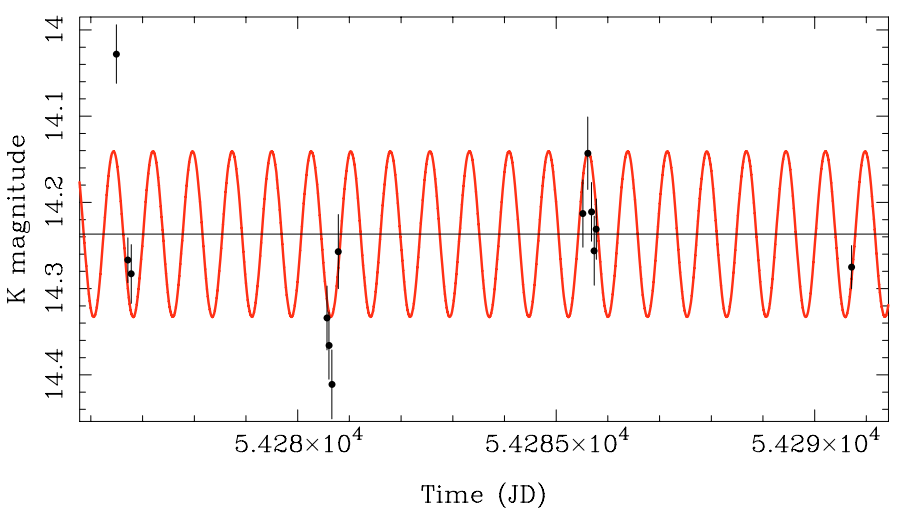

Time (JD)

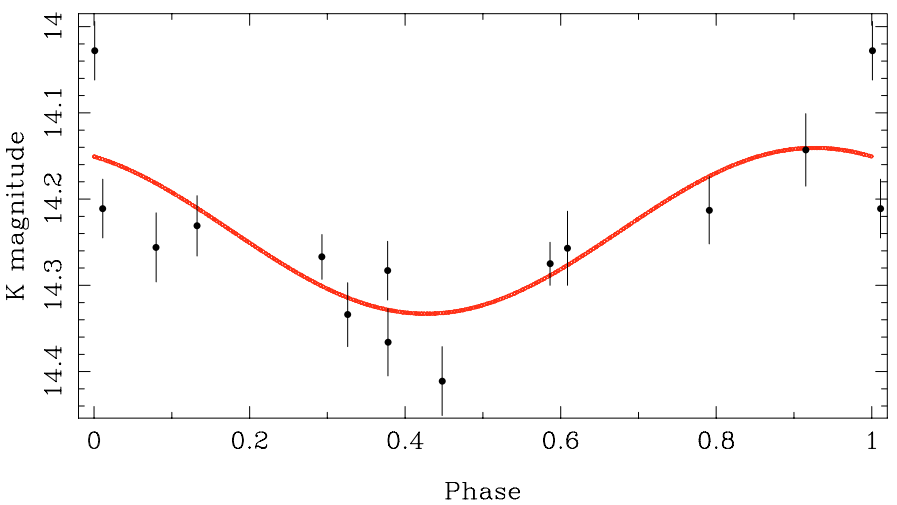

bul39_5385
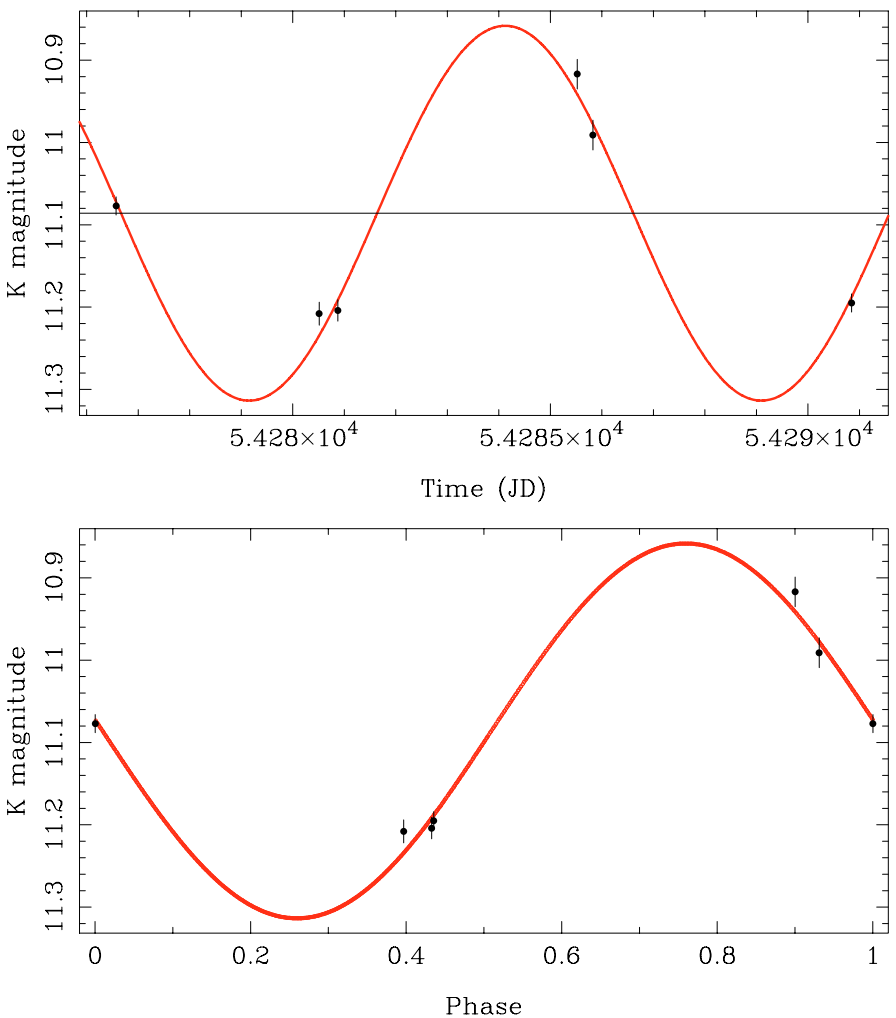

Fig. 3. The $K$-band light curves of two P2C. The top panel shows the magnitude versus time, the bottom panel the phased light curve.

to $7.99 \pm 0.09 \mathrm{kpc}$. The error takes into account the precision in our $\mathrm{ZP}$ and the error in the $\mathrm{ZP}$ of the M06 relation.

Even adding in quadrature an additional 0.015 mag to the error on the photometry (allowing for an underestimate of the adapted error on the transformation from instrumental magnitude to the 2MASS system) and doubling the error on the $A_{\mathrm{K}}$ value (with a minimum error of 0.03 ) increases the formal error bar on the derived ZP only to $0.025 \mathrm{mag}$ and the error on the DM to $0.03(0.12 \mathrm{kpc})$.

\subsection{P2C: the Wesenheit-index PL-relation}

An alternative method of deriving the distance is to use a reddening-free Wesenheit index. The most appropriate one is $W I K=I-\alpha(I-K)$, as the $I$-band magnitudes are available from OGLE. Unfortunately the available $I$-band data (Pritzl et al. 2003) for stars with $K$-band data in M06 is insufficient for deriving an empirical relation, but one can use theoretical $P L$-relations as derived in several bands by Di Criscienzo (2007). These theoretical relations are in good agreement with observations. The slope of the theoretical $I$-band $P L$-relation, $-2.10 \pm 0.06$, agrees with the observed one of $-2.03 \pm 0.03$ (Pritzl et al. 2003), while in the $K$-band the theoretical slope of $-2.38 \pm 0.02$ is in good agreement with the observed $-2.41 \pm 0.02$ in M06. For an assumed mixing length parameter of 1.5 , one needs to adopt a fiducial $[\mathrm{Fe} / \mathrm{H}]$ value of -1.63 in the theoretical $P L$-relation to obtain the observed $I$ magnitude at a typical period of $\log P=0.5$ (Pritzl et al. 2003), and similarly a fiducial $[\mathrm{Fe} / \mathrm{H}]$ of -2.55 to obtain the observed $K$ magnitude at $\log P=0.5$ (M06).
Depending on the coefficient $\alpha$ in the Wesenheit-relation (Di Criscienzo, private comm.) the theoretical relation is, $W I K=(-1.13 \pm 0.02)-(2.53 \pm 0.04) \log P+(0.06 \pm 0.01)[\mathrm{Fe} / \mathrm{H}]$ for $\alpha_{1}=1.252$ (standard reddening law) or $(-1.17 \pm 0.02)-$ $(2.57 \pm 0.04) \log P+(0.06 \pm 0.01)[\mathrm{Fe} / \mathrm{H}]$ for $\alpha_{2}=1.316$ (allowing for anomalous reddening in $I$, see Sumi 2004). For an $[\mathrm{Fe} / \mathrm{H}]$ value of $-2.0 \pm 0.4$, one expects ZPs of $-1.25 \pm 0.04\left(\alpha_{1}\right)$ and $-1.29 \pm 0.04\left(\alpha_{2}\right)$

A potential complication is that the theoretical relation is derived for the Bessell \& Brett (1988) photometric system, while in particular the OGLE $I$-filter is slightly non-standard. An appropriate transformation is applied to both the 2MASS $K$ and OGLE $I$ to the Bessell \& Brett system. It turns out, however, that for the typical $(V-I)$ colours of the $\mathrm{P} 2 \mathrm{C}$ in the observed fields and the particular value of the slope in the Wesenheit-index the corrections largely cancel, and the effect on the derived ZP is minimal.

For $\alpha_{1}$ the derived slope from the observations is $-2.25 \pm$ 0.17 , for $\alpha_{2}-2.27 \pm 0.18$. The agreement between the observed and theoretical slopes is not as good (about 1.6-1.7 $\sigma$ deviation) as the one in the $K$-band (only $1.3 \sigma$ deviation).

When the slope is fixed to the theoretical value, one obtains ZPs of $13.19 \pm 0.01$ and $13.04 \pm 0.01$, respectively, based on a Monte-Carlo simulation. Depending on the reddening law adopted, one obtains a DM of about 14.44 (7.73 kpc), or 14.33 $(7.35 \mathrm{kpc})$ with an internal error bar of $0.04 \mathrm{mag}(0.14 \mathrm{kpc})$.

\subsection{RR Lyrae}

Sollima et al. (2006) derive an empirical PLK-relation based on 15 GC and the LMC cluster Reticulum, which for a metallicity 
Table 1. The sample of Cepheids, ordered by period.

\begin{tabular}{|c|c|c|c|c|c|c|c|c|c|c|c|c|c|c|c|}
\hline Name & $\begin{array}{c}\text { RA } \\
\text { (J2000) }\end{array}$ & $\begin{array}{c}\text { Dec } \\
(\mathrm{J} 2000)\end{array}$ & $\begin{array}{r}\text { Period } \\
\text { (days) }\end{array}$ & $l$ & $b$ & $A_{\mathrm{K}}^{(1)}$ & $\sigma$ & $n$ & $A_{\mathrm{K}}^{(2)}$ & $A_{\mathrm{K}}^{(3)}$ & $\sigma$ & $n$ & $K$ & $\sigma$ & $n$ \\
\hline bul24_1609 & 175344.41 & -325714.0 & 0.76522 & 357.44 & -3.56 & 0.284 & 0.018 & 6 & 0.302 & - & $\begin{array}{ll}- \\
-\end{array}$ & - & 14.237 & 0.010 & 13 \\
\hline bul214.2_135 & 175735.12 & -282611.6 & 0.82684 & 1.77 & -2.00 & 0.317 & 0.028 & 5 & - & 0.354 & 0.011 & 6 & 13.805 & 0.049 & 5 \\
\hline bul214.6_53 & 1756 & -281402.3 & 3088 & 1.80 & -1.65 & 0.352 & 0.005 & 3 & - & - & - & - & 14.118 & 0.057 & 6 \\
\hline bul39_2239 & 1756 & -29 & & 0.33 & -2.46 & 0.275 & 0.026 & 6 & 0.316 & 0.343 & 0.023 & 6 & 13.836 & 0.023 & 6 \\
\hline bul22_? & 17563 & -3036 & 0.93110 & 359.77 & -2.89 & 0.330 & 0.051 & 6 & 0.329 & - & - & - & 13.583 & 0.013 & 9 \\
\hline 62 & 6.92 & -285 & 0.939 & 1.82 & -3.07 & 0.193 & 0.019 & 6 & 0.217 & 0.237 & 0.021 & 3 & 13.925 & 0.010 & 8 \\
\hline 1 & 3.13 & -27312 & 1.09776 & 359.97 & 2.71 & 0.492 & 0.045 & 6 & 0.440 & - & - & - & 13.629 & 0.007 & 13 \\
\hline $3.7 \_46320$ & 7515 & -31563 & 1.21123 & 358.12 & -2.71 & 0.433 & 0.078 & 4 & - & - & - & - & 12.573 & 0.010 & 11 \\
\hline -110/ & 180147.33 & -29073 & 1.33916 & 1.63 & -3.14 & 0.215 & 0.016 & 4 & 0.229 & 0.233 & 0.015 & 4 & 12.126 & 0.006 & 11 \\
\hline 5.7_107864 & 175854.55 & -28 2130.8 & 1.40015 & 1.98 & -2.22 & 0.282 & 0.035 & 6 & - & 0.278 & 0.011 & 4 & 13.088 & 0.009 & 11 \\
\hline & 175327.82 & -301955.3 & 1.48412 & 359.68 & -2.18 & 0.323 & 0.049 & 5 & 0.347 & - & - & - & 14.255 & 0.022 & 8 \\
\hline bul5_3719 & 175021.49 & -295427.5 & 1.50105 & 359.70 & -1.39 & 0.310 & 0.023 & 4 & 0.688 & - & - & - & 13.304 & 0.028 & 7 \\
\hline bul32_2167 & 180256.05 & -284039.2 & 1.50523 & 2.14 & -3.14 & 0.178 & 0.012 & 4 & 0.193 & 0.212 & 0.011 & 5 & 13.351 & 0.008 & 11 \\
\hline bul4_170 & 175438.21 & -30 1041.8 & 1.53185 & 359.94 & -2.32 & 0.278 & 0.027 & 5 & 0.311 & - & - & - & 13.027 & 0.010 & 10 \\
\hline ul44_5324 & 174930.95 & -295058.2 & 1.55140 & 359.66 & -1.20 & 0.313 & 0.039 & 5 & 0.720 & - & - & - & 449 & 0.011 & 8 \\
\hline 38.1_11087 & 175930.71 & -30 1959.7 & & 0.33 & -3.31 & 0.2 & 0.045 & 5 & - & - & - & - & & 18 & 9 \\
\hline & 17595 & -2856 & & 1.58 & -2.69 & 0.198 & 0.019 & 5 & - & 0.219 & 0.014 & 5 & & .017 & 9 \\
\hline & & -30( & & 1.04 & -3.92 & 0.1 & 0.019 & 6 & 0.220 & 0.169 & 0.009 & 5 & & 0.018 & 9 \\
\hline & & -31 & & 359.13 & -3.68 & 0.3 & 0.009 & 4 & 0.324 & - & - & - & & 0.007 & 10 \\
\hline & $1758 \mathrm{C}$ & -285956.6 & 33010 & 1.33 & -2.37 & 0.234 & 0.031 & 4 & 0.302 & 0.312 & .033 & 5 & 12.690 & 0.024 & 9 \\
\hline & 93 & -291 & & 1.27 & -2.63 & 0.223 & 0.038 & 6 & 0.233 & 0.219 & 0.004 & 5 & 12.661 & 0.006 & 9 \\
\hline 841 & 17514 & -324141.3 & 3.20082 & 357.45 & -3.06 & 0.346 & 0.028 & 5 & 0.318 & - & - & - & 12.196 & 0.008 & 9 \\
\hline & 75406.61 & -291622.1 & 3.51336 & 0.66 & -1.76 & 0.295 & 0.025 & 5 & 0.311 & - & - & - & 12.449 & 0.012 & 6 \\
\hline bu & 5.52 & -295731.0 & 3.54254 & 0.16 & -2.26 & 0.271 & 0.022 & 6 & 0.311 & 0.286 & 0.010 & 5 & 13.267 & 0.112 & 4 \\
\hline bul3 & 175512.35 & -300724.1 & 3.65017 & 0.05 & -2.40 & 0.268 & 0.023 & 4 & 0.316 & - & - & - & 12.281 & 0.007 & 8 \\
\hline bul1 & 175340.70 & -285647.0 & 3.82791 & 0.90 & -1.52 & 0.315 & 0.024 & 4 & - & - & - & - & 12.101 & 0.014 & 4 \\
\hline bul 2 & 17564 & -310707.3 & 4.49944 & 359.36 & -3.19 & 0.270 & 0.031 & 5 & 0.329 & - & - & - & 12.415 & 0.035 & 7 \\
\hline bul3_1755 & 175334.75 & -30 1239.7 & 4.57875 & 359.80 & -2.14 & 0.318 & 0.046 & 6 & 0.347 & - & - & - & 12.613 & 0.055 & 7 \\
\hline bul38_3260 & 180132.68 & -294911.5 & 5.53097 & 1.00 & -3.44 & 0.214 & 0.027 & 7 & 0.220 & 0.206 & 0.018 & 4 & 12.175 & 0.121 & 5 \\
\hline bul180.5_151030 & 175157.55 & $\begin{array}{llll}-30 & 21 & 39.2\end{array}$ & 6.89131 & 359.49 & -1.91 & 0.381 & 0.042 & 5 & - & - & - & - & 11.601 & 0.007 & 6 \\
\hline bul20_3867 & 175855.95 & -284319.5 & 7.13700 & 1.67 & -2.40 & 0.233 & 0.031 & 4 & 0.233 & 0.243 & 0.012 & 5 & 11.639 & 0.007 & 6 \\
\hline bul2_1657 & 180419.87 & -290244.2 & & 1.97 & -3.59 & 0.174 & 0.007 & 6 & 0.186 & 0.189 & 0.014 & 4 & 11.464 & 0.007 & 6 \\
\hline & & -330 & & 357.37 & -3.76 & 0.275 & 0.020 & 6 & 0.281 & - & - & - & 11.775 & 0.006 & 6 \\
\hline-2024 & 175058.92 & -3308 & & 356.98 & -3.16 & 0.344 & 0.022 & 6 & 0.353 & - & - & - & 11.999 & 0.006 & 6 \\
\hline 4.5_169880 & 175637.75 & -280128 & & 2.02 & -1.61 & 0.367 & 0.050 & 5 & - & 0.462 & 0.017 & 5 & 12.180 & 0.007 & 5 \\
\hline & 180844.36 & -32131 & 9.52109 & 359.64 & -5.95 & 0.149 & 0.004 & 4 & 0.160 & - & - & - & 11.220 & 0.006 & 6 \\
\hline & & -29313 & & 0.58 & -2.13 & 0.2 & 0.025 & 4 & 0.316 & 0.323 & 0.006 & 4 & 11.086 & 0.007 & 6 \\
\hline & & & & 2.74 & 2.94 & & & 7 & - & - & - & - & 14.200 & 0.018 & 5 \\
\hline bul333.2_25311 & 173634.05 & -271808.3 & 14.89102 & 0.33 & 2.56 & 0.449 & 0.034 & 4 & - & - & - & & 10.856 & 0.008 & 5 \\
\hline
\end{tabular}

Notes: (1) Reddening from Marshall et al. (2006). (2) Reddening based on Sumi (2004). (3) Reddening based on Popowski et al. (2003).

of $[\mathrm{Fe} / \mathrm{H}]=-1.0$ (the average metallicity of RR Lyrae in the GB, see Walker $\&$ Terndrup 1991), reads $M_{\mathrm{K}}=(-2.38 \pm 0.04) \log P-$ $(1.13 \pm 0.13)$, as calibrated against the trigonometric parallax of RR Lyra (Benedict et al. 2002). Fig. 6)

Removing five bright outliers, the PL-relation becomes (cf.

$$
\begin{aligned}
K_{0}= & (-1.36 \pm 0.49) \log P+(13.63 \pm 0.17) \\
& +(+0.019 \pm 0.021) l-(0.025 \pm 0.043) b .
\end{aligned}
$$

The error in the derived slope is large but formally agrees within $2 \sigma$ with the empirical slope by Sollima et al. For a fixed slope of -2.38 , the ZP becomes $13.39 \pm 0.13$ (based on a Monte-Carlo simulation), resulting in a DM of $14.52 \pm 0.18$ $(8.0 \pm 0.7 \mathrm{kpc})$.

\section{Discussion}

The $P L$-relation has been derived in the $K$-band of $\mathrm{P} 2 \mathrm{C}$ in the GB. The slope is found to agree with the one derived by M06. Fixing the slope to their more accurate value implies a DM to the GC of 14.51 with a formal error bar of 0.03 .
There is also the systematic error bar to consider. M06 presented $J H K$ period-luminosity relations based on $46 \mathrm{P} 2 \mathrm{C}$ with periods between 1.2 and 80 days in 26 Galactic globular clusters (GGCs). For the absolute magnitude scale, they adopted a relation between absolute $V$-magnitude of the horizontal branch and metallicity (Gratton et al. 2003), which in turn is calibrated using main-sequence fitting to three GGCs. This calibration im-

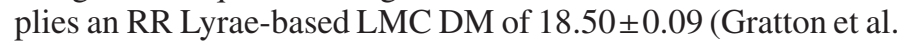
2003). M06 show that the DM based on P2C in the LMC and their $K$-band $P L$-relation is also compatible with 18.5.

They also show that there is no significant trend with metallicity over the range $-2.2 \lesssim[\mathrm{Fe} / \mathrm{H}] \lesssim-0.5$, in agreement with theoretical predictions (Bono et al. 1997, 2003; Di Criscienzo et al. 2007) and indicating that this $K$-band $P L$-relation should be applicable for GC P2C as well. The metallicity of the P2C in the GB is unknown, but that of RR Lyrae is estimated to be on average $[\mathrm{Fe} / \mathrm{H}]=-1.0$ (Walker \& Terndrup 1991). Any difference between that metallicity and the mean metallicity of about $[\mathrm{Fe} / \mathrm{H}]=-1.5$ of the GCs in M06 would result in an uncertainty in the ZP of $\lesssim 0.03$ mag. Di Criscienzo et al. (2007) show that adopting a different $M_{\mathrm{V}}-[\mathrm{Fe} / \mathrm{H}]$ relation has a negligible effect 
Table 2. The sample of RR Lyrae stars, ordered by period.

\begin{tabular}{|c|c|c|c|c|c|c|c|c|}
\hline Name & $\begin{array}{c}\text { RA } \\
(\mathrm{J} 2000)\end{array}$ & $\begin{array}{c}\text { Dec } \\
(\mathrm{J} 2000)\end{array}$ & $\begin{array}{r}\text { Period } \\
\text { (days) }\end{array}$ & P2C field & $\bar{K}$ & $\sigma$ & $n$ & Remark \\
\hline bul40_259253 & 17.85087 & -33.1519 & 0.39951 & bul40_2524 & 14.899 & 0.021 & 5 & \\
\hline bul39_15393 & 17.92076 & -30.1590 & 0.43787 & bul39_616 & 12.685 & 0.017 & 6 & outlier \\
\hline bul31_680405 & 18.04767 & -28.6609 & 0.43940 & bul32_2167 & 14.536 & 0.011 & 11 & \\
\hline bul45_245148 & 18.05646 & -30.0464 & 0.44022 & bul45_1189 & 14.410 & 0.010 & 8 & \\
\hline bul20_43721 & 17.98309 & -29.1750 & 0.45910 & bul20_961 & 14.399 & 0.013 & 8 & \\
\hline bul24_369931 & 17.89280 & -32.9691 & 0.47306 & bul24_1609 & 13.951 & 0.008 & 12 & \\
\hline bul45_256663 & 18.05978 & -30.0235 & 0.47416 & bul45_1189 & 14.421 & 0.018 & 7 & \\
\hline bul3_239578 & 17.89180 & -30.3038 & 0.48308 & bul3_791 & 14.605 & 0.082 & 4 & \\
\hline bul22_133009 & 17.93951 & -30.6361 & 0.48700 & bul22_3993 & 14.331 & 0.011 & 9 & \\
\hline bul44_201914 & 17.82289 & -29.8616 & 0.48806 & bul44_5324 & 11.237 & 0.006 & 6 & outlier \\
\hline bul20_44361 & 17.98202 & -29.1714 & 0.48950 & bul20_961 & 14.994 & 0.021 & 6 & \\
\hline bul4_207615 & 17.90976 & -30.1619 & 0.50215 & bul4_170 & 14.405 & 0.028 & 7 & \\
\hline bul31_23420 & 18.03285 & -28.9437 & 0.50610 & bul31_662 & 12.923 & 0.020 & 5 & outlier \\
\hline bul22_219909 & 17.94488 & -31.1046 & 0.52411 & bul22_815 & 12.718 & 0.007 & 6 & outlier \\
\hline bul39_156007 & 17.92021 & -29.5568 & 0.52499 & bul39_5385 & 14.119 & 0.026 & 4 & \\
\hline bul34_159387 & 17.96703 & -28.9742 & 0.54158 & bul34_4631 & 14.437 & 0.017 & 7 & \\
\hline bul40_107313 & 17.84697 & -33.1318 & 0.54215 & bul40_2524 & 14.430 & 0.024 & 5 & \\
\hline bul4_451052 & 17.91352 & -29.9600 & 0.54390 & bul4_2323 & 14.185 & 0.013 & 8 & \\
\hline bul5_261524 & 17.84023 & -29.8885 & 0.55190 & bul5_3719 & 14.320 & 0.027 & 8 & \\
\hline bul22_402472 & 17.94676 & -31.0980 & 0.55482 & bul22_815 & 14.408 & 0.014 & 6 & \\
\hline bul4_439650 & 17.91289 & -29.9957 & 0.55603 & bul4_2323 & 14.419 & 0.011 & 7 & \\
\hline bul32_87588 & 18.05029 & -28.6886 & 0.57740 & bul32_2167 & 13.994 & 0.014 & 9 & \\
\hline bul22_144794 & 17.94068 & -30.6044 & 0.57910 & bul22_3993 & 14.364 & 0.016 & 9 & \\
\hline bul25_187102 & 17.90468 & -33.1508 & 0.57918 & bul25_693 & 14.244 & 0.015 & 5 & \\
\hline bul22_144296 & 17.94163 & -30.5937 & 0.58562 & bul22_3993 & 14.261 & 0.012 & 9 & \\
\hline bul43_130470 & 17.58358 & -27.5280 & 0.59590 & bul43_351 & 14.246 & 0.013 & 10 & \\
\hline bul22_333396 & 17.94312 & -30.5768 & 0.61422 & bul22_3993 & 14.525 & 0.036 & 4 & \\
\hline bul24_527005 & 17.89388 & -32.9262 & 0.61678 & bul24_1609 & 14.356 & 0.011 & 10 & \\
\hline bul3_250926 & 17.89106 & -30.2063 & 0.63179 & bul3_1755 & 13.017 & 0.013 & 6 & outlier \\
\hline bul34_159754 & 17.96594 & -29.0003 & 0.63539 & bul34_4631 & 14.066 & 0.016 & 9 & \\
\hline bul45_245339 & 18.05951 & -30.0290 & 0.63597 & bul45_1189 & 14.034 & 0.045 & 6 & \\
\hline bul34_145186 & 17.96658 & -29.0164 & 0.66322 & bul34_4631 & 14.193 & 0.025 & 4 & \\
\hline bul30_604974 & 18.03144 & -29.1517 & 0.67131 & bul30_1107 & 14.361 & 0.025 & 8 & \\
\hline bul34_389919 & 17.96842 & -29.0303 & 0.67304 & bul34_4631 & 14.334 & 0.016 & 7 & \\
\hline bul39_15358 & 17.92118 & -30.1017 & 0.69769 & bul39_616 & 14.323 & 0.040 & 5 & \\
\hline bul24_517291 & 17.89567 & -32.9539 & 0.76522 & bul24_1609 & 14.237 & 0.010 & 13 & \\
\hline bul4_207388 & 17.90973 & -30.1789 & 0.78642 & bul4_170 & 14.090 & 0.015 & 6 & \\
\hline
\end{tabular}

on the derived slope of the NIR PL-relations. The ZP in the calibrating relation by Gratton et al. has a formal error of 0.07 and this has to be considered as a source of systematic uncertainty in the derived distance.

There is an other source of (random) error to consider, namely how representative this particular set of 39 stars (minus the one outlier) that defines the $P L$-relation is in view of the fact that they scatter along the line-of-sight due to the intrinsic depth of the GB. To simulate this, additional Monte-Carlo simulations were carried out. Random samples of 38 stars were selected from the original sample, and the $P L$-relation re-derived. The dispersion in the ZP is about $0.11 \mathrm{mag}$. This is likely a slight overestimate, as in this approach the randomly drawn samples do not necessarily have the wide spread in period that the true sample was selected to have.

The DM to the GC we derived based on the $\mathrm{P} 2 \mathrm{C}$ is $14.51 \pm$ 0.12 (random) \pm 0.07 (syst). The random error could be improved further by observing additional systems when the full OGLE-III database becomes available.

Based on the serendipitously observed RR Lyrae stars in the field, a DM of $14.52 \pm 0.18$ was derived. The error bar is for $50 \%$ due to the uncertainty in the adopted absolute magnitude of RR Lyra itself (Sollima et al.). Their PL-relation led to an LMC distance of $18.54 \pm 0.15$. If instead we were to assume the LMC distance to be 18.50 (to be consistent with the $\mathrm{P} 2 \mathrm{C}$ calibration), then we would find a DM of $14.48 \pm 0.13$ (random) \pm 0.07 (syst), where the systematic error comes from the uncertainty in the ZP of the observed LMC PL-relation. As for the P2C sample, there is an additional $0.11 \mathrm{mag}$ systematic uncertainty due to the limited sample size. The final DM to the GC based on the $K$-band RR Lyrae stars is $14.48 \pm 0.17$ (random) \pm 0.07 (syst). As the two distance estimates are derived independently, they can be averaged and the best empirical estimate of the DM to the GC based on the current data is $14.50 \pm 0.10$ (random) \pm 0.07 (syst).

The theoretical WIK relation gives a formal result of 14.44 (or 14.33 with anomalous reddening) with an internal error bar of $0.04 \mathrm{mag}$. A random error of 0.11 has to be added to this, due to the limited sample. As the theoretical relation is tied to the observed relations of M06 a similar systematic error bar of 0.07 has to be considered.

Although within the error bar of the purely empirical results, this brings up the question of reddening and the reddening law. An additional absorption in $K$ of 0.1 mag would bring all three methods into very good agreement. On the other hand, the reddening estimates listed in Table 1 are in excellent agreement and are based on $(J-K)$ colours (Marschall et al. 2006), $(V-I)$ (Sumi 2004), and $(V-R)$ (Popowski et al. 2003). If the absorption in $K$ were underestimated, it would also imply an underestimate of 

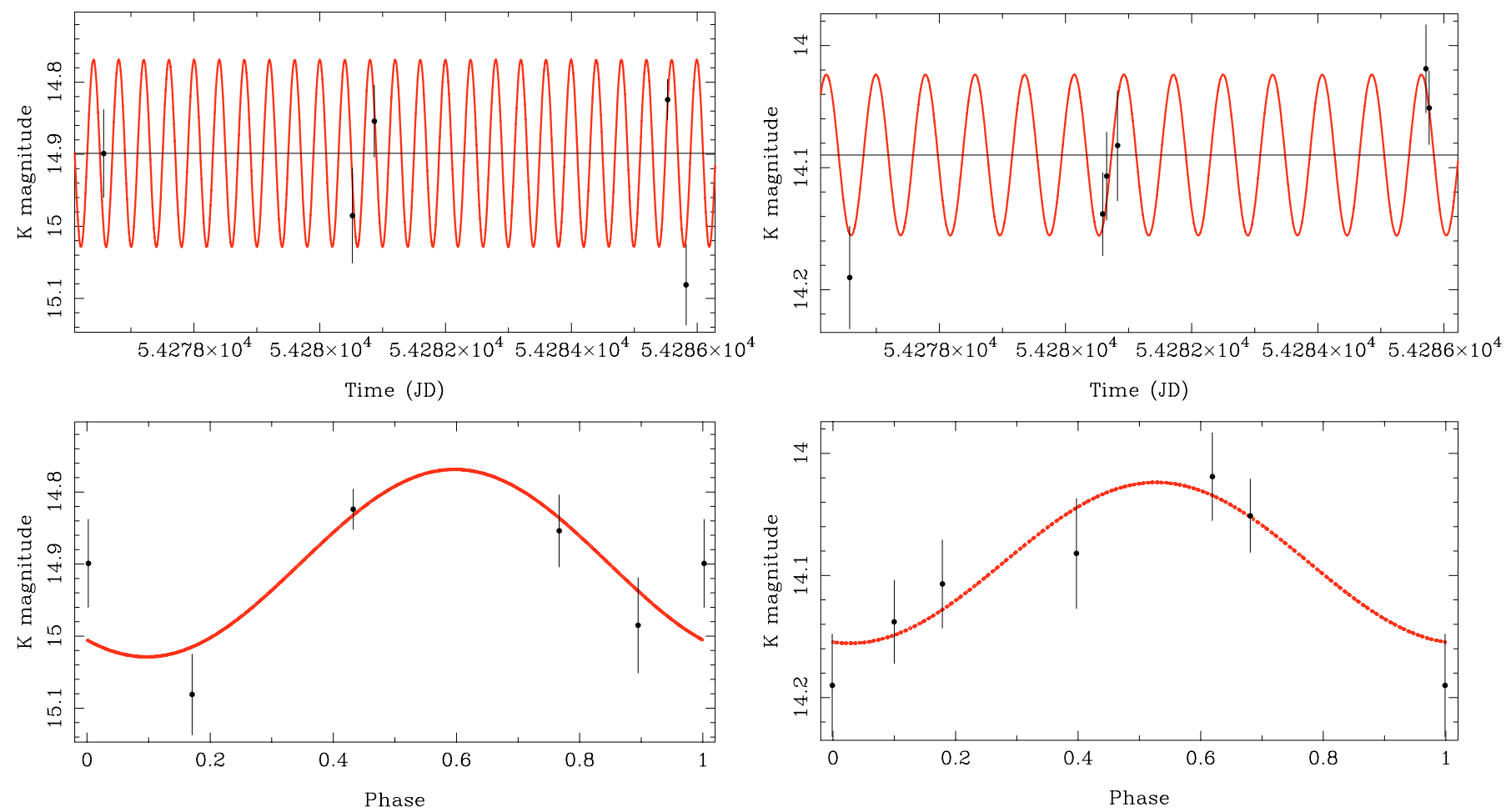

Fig. 4. The $K$-band light curves of two RR Lyrae. The top panel shows the magnitude versus time, the bottom panel the phased light curve.

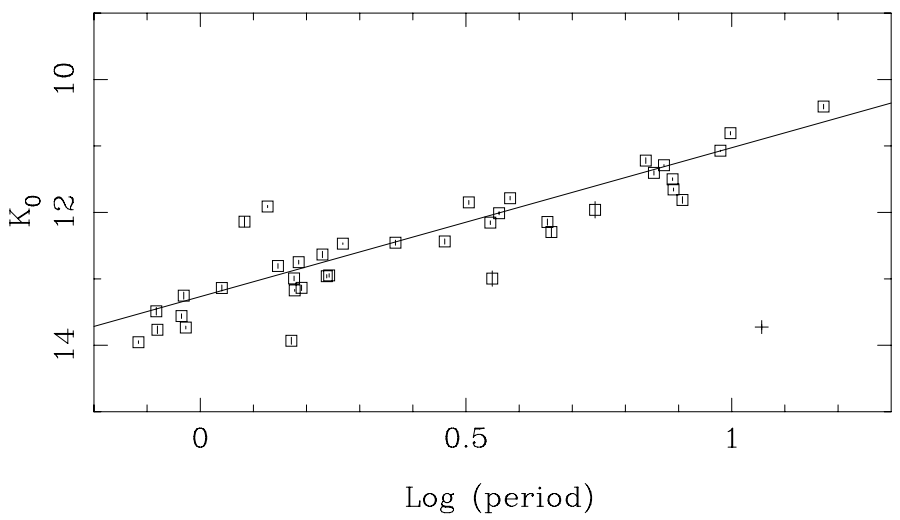

Fig. 5. The $K$-band PL-relation for $\mathrm{P} 2 \mathrm{C}$ in the $\mathrm{GB}$. The line is a best fit excluding the cross.

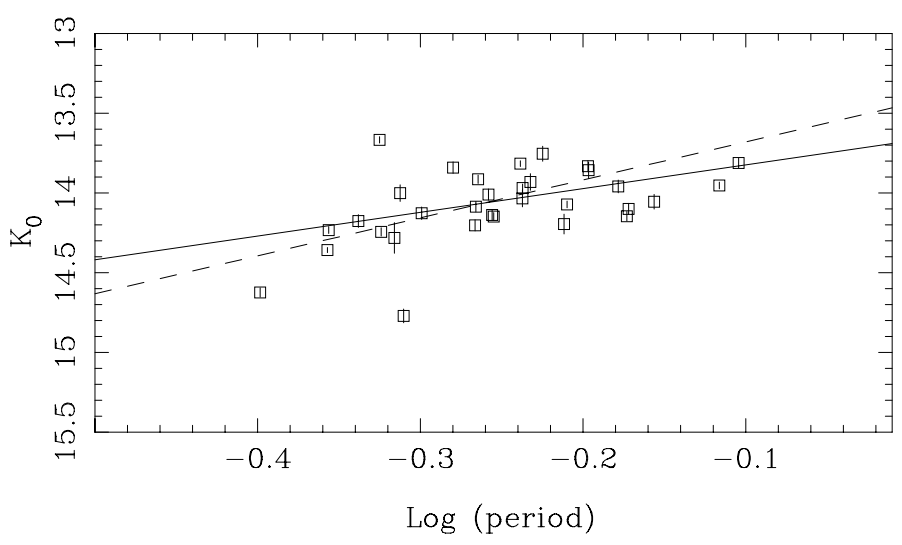

Fig. 6. The $K$-band PL-relation for the RR Lyrae stars in the GB. The line is a best fit excluding the outliers labelled in Table 2 which fall outside the plot. The dotted line is the fit for a fixed slope of -2.38 . the reddening in the other maps or a significantly higher selective reddening $A_{\mathrm{K}} / A_{\mathrm{V}} \sim 0.16$ instead of 0.12 .

A final remark is that independent distances to some of these P2C may be obtained using surface-brightness relations (e.g. Groenewegen 2004) and the Baade-Wesselink technique. This would require better-sampled $K$-band light curves than were needed for the present study and well-sampled radial velocity curves. Although observationally expensive, it would give an improved understanding on the systematic error in the present analysis.

Acknowledgements. M.G. would like to thank ESO astronomers Valentin Ivanov and Alessandro Ederoclite for their support at the telescope and discussion of the SOFI pipeline, and Evelien Vanhollebeke for the introduction to the DoPhot package. The first draft of this paper was written when M.G. was a shortterm visitor at the Max-Planck Institut für Astrophysik, Garching. AU was partly supported by the Polish MNiSW grant N20303032/4275. G.B. thanks PRIN/INAF 2006 (PI: F. Ferraro) for partial support. This publication makes use of data products from the Two Micron All Sky Survey, which is a joint project of the University of Massachusetts and the Infrared Processing and Analysis Center/California Institute of Technology, funded by the National Aeronautics and Space Administration and the National Science Foundation.

\section{References}

Babusiaux, C., \& Gilmore, G. 2005, MNRAS, 358, 1309 Bessell, M. S., \& Brett, J. M. 1988, PASP, 100, 1134 Benedict, G. F., McArthur, B. E., Fredrick, L., et al. 2002, ApJ, 581, 115

Bono, G., Caputo, F., \& Santolamazza, P. 1997, A\&A, 317, 171

Bono, G., Caputo, F., Castellani, V., et al. 2003, MNRAS, 344, 1097

Cardelli, J. A., Clayton, G. C., \& Mathis, J. S. 1989, ApJ, 345, 245

Carney, B. W., Fulbright, J. P., Terndrup, D. M., et al. 1995, AJ, 110, 1674

Catchpole, R., Whitelock, P. A., Feast, M. W., et al. 1999, IAU Symp., 192 (ASP), 89

Collinge, M. J., Sumi, T., \& Fabrycky, D. 2006, ApJ, 651, 197

Di Criscienzo, M., Caputo, F., Marconi, M., \& Cassisi, S. 2007, A\&A, 471, 893

Eisenhauer, F., Schödel, R., Genzel, R., et al. 2003, ApJ, 597, L121

Eisenhauer, F., Genzel, R., Alexander, T., et al. 2005, ApJ, 628, 246 
Feast, M. W., \& Whitelock, P. A. 1997, MNRAS, 291, 683

Fernley, J. A., Longmore, A. J., Jameson, R. F., Watson, F. G., \& Wesselink, T. 1987, MNRAS, 226, 927

Gratton, R. G., Bragaglia, A., Carretta, E., et al. 2003, A\&A, 408, 529

Groenewegen, M. A. T. 2004, MNRAS, 353, 903

Groenewegen, M. A. T., \& Blommaert, J. A. D. L. 2005, A\&A, 443, 143

Kubiak, M., \& Udalski, A. 2003, AcA, 53, 117 (KU)

Marshall, D. J., Robin, A. C., Reylé, C., Schultheis, M., \& Picaud, S. 2006, A\&A, 453, 635

Matsunaga, N., Fukushi, H., \& Nakada, Y., et al. 2006, MNRAS, 370, 1979 (M06)

McNamara, D. H., Madsen, J. B., Barnes, J., \& Ericksen, B. F. 2000, PASP, 112 , 202
Nishiyama, S., Nagata, T., Sata, K., et al. 2006, ApJ, 647, 1093

Paczyński, B., \& Stanek, C. 1998, ApJ, 494, L129

Popowski, P., Cook, K. H., \& Becker, A. C. 2003, AJ, 126, 2910

Pritzl, B. J., Smith, H. A., Stetson, P. B., et al. 2003, AJ, 126, 1381

Reid, M. J. 1993, ARA\&A, 31, 345

Schechter, P. L., Mateo, M. L., \& Saha, A. 1993, PASP, 105, 1342

Sollima, A., Cacciari, C., \& Valenti, E. 2006, MNRAS, 372, 675

Sumi, T. 2004, MNRAS, 349, 193

Vanhollebeke, E., Groenewegen, M. A. T., \& Girardi, L. 2008, A\&A, submitted Walker, A. R., \& Terndrup, D. M. 1991, ApJ, 378, 119

Zucker, S., Alexander, T., Gillessen, S., Eisenhauer, F., \& Genzel, R. 2006, ApJ, 639, L21 